\title{
Physical Literacy in the Culture of Physical Education in Elementary Schools: Indonesian Perspectives
}

\author{
Johan Irmansyah", ${ }^{1,}$ Ermawan Susanto², Ria Lumintuarso², FX. Sugiyanto², Ahmad Syarif ${ }^{3}$, \\ Hermansyah $^{1}$

\begin{abstract}
${ }^{1}$ Department of Sport and Health Education, Faculty of Sports Science and Public Health, Universitas Pendidikan Mandalika, Mataram, 83125, Indonesia

${ }^{2}$ Department of Sport Education, Faculty of Sports Science, Universitas Negeri Yogyakarta, Yogyakarta, 55281, Indonesia

${ }^{3}$ Graduate Schools, Universitas Negeri Yogyakarta, Yogyakarta, 55281, Indonesia
\end{abstract}

Received May 31, 2021; Revised July 12, 2021; Accepted August 22, 2021

\section{Cite This Paper in the following Citation Styles}

(a): [1] Johan Irmansyah, Ermawan Susanto, Ria Lumintuarso, FX. Sugiyanto, Ahmad Syarif, Hermansyah, "Physical Literacy in the Culture of Physical Education in Elementary Schools: Indonesian Perspectives, "International Journal of Human Movement and Sports Sciences, Vol. 9, No. 5, pp. 929 - 939, 2021. DOI: 10.13189/saj.2021.090514.

(b): Johan Irmansyah, Ermawan Susanto, Ria Lumintuarso, FX. Sugiyanto, Ermawan Susanto, Ahmad Syarif, Hermansyah (2021). Physical Literacy in the Culture of Physical Education in Elementary Schools: Indonesian Perspectives. International Journal of Human Movement and Sports Sciences, 9(5), 929 - 939. DOI: 10.13189/saj.2021.090514.

Copyright $\odot 2021$ by authors, all rights reserved. Authors agree that this article remains permanently open access under the terms of the Creative Commons Attribution License 4.0 International License

\begin{abstract}
Several countries around the world have promoted, advocated, and developed the concept of physical literacy. The concept of physical literacy is being believed to offer alternative solutions in the future as an effort to overcome global problems related to the lack of interest in individuals in performing physical activity. This research aims to develop a model of physical literacy in the culture of physical education in elementary schools, reviewed from the perspective of West Nusa Tenggara and the Special Region of Yogyakarta, Indonesia. The research method uses a mixed method with sequential exploratory strategies. The research procedure consists of three phases; Phase 1. Design of Physical Literacy; Phase 2. Expert Consensus ( $\mathrm{n}=8)$; and Phase 3. Practitioners Assessment $(n=12)$. The results of the study in Phase 1 , which was analyzed qualitatively, produced three main themes, namely; (a) theoretical foundation, (b) construction of physical literacy, and (c) operationalization of physical literacy. In Phase 2 and 3, which were analyzed quantitatively, experts and practitioners reached a statement of agreement regarding the physical literacy model which was developed, with a percentage value of 86.01\% (Phase 2) and $91.84 \%$ (Phase 3). These results show that the physical literacy model developed is in accordance with the culture of physical education in elementary schools, and can be implemented in the
\end{abstract}

physical education learning process.

Keywords Elementary Schools, Expert Consensus, Physical Education, Physical Literacy, Teacher Assessment

\section{Introduction}

Physical literacy is a term that is the subject of debate in several countries related to philosophical assumptions, concepts, definitions, and assessments in operationalizing physical literacy [1]. In recent years, international attention has been increased by the integration of physical literacy in the policies or regulations of physical education, sports, recreation, and health [2], thus physical literacy is expected to affect individual physical activity levels, physical fitness, motor skills, decreased obesity, and prevention of non-communicable diseases [3]. Physical literacy is also considered relevant to various domains, such as affective, cognitive, physical competence, and behavior. However, in its rapid development, the formulation of the concept and definition of physical literacy that became the basis for the promotion and development of physical literacy, there are still differences 
in some countries [4]. This situation can cause confusion from researchers in conducting scientific reviews and research, as well as confusion of physical education teachers in conducting implementation and assessment of physical literacy.

Whitehead [5], [6] underlies the concept of physical literacy in the embodiment of man as a living individual, which is a fundamental aspect of human nature, thus affecting many aspects of life, such as; development of self-realization, confidence, positive self-esteem, and great potential to improve quality of life through the promotion and development of physical literacy. The concept of physical literacy should also provide a structure in which the meaning of human embodiment can be identified, understood, and appreciated, not only used as an instrument [7]. This concept is what makes physical literacy certainly being believed to offer alternative solutions in the future as an effort to overcome global problems related to the lack of interest in individuals in performing physical activity [8], [9]. Furthermore, Whitehead [10] also described physical literacy as "motivation, confidence, physical competence, knowledge and understanding to value and take responsibility for engagement in physical activities for life". This definition of physical literacy, has been used and adopted up to $(70 \%)$ by most papers in scientific research and international organizations [11]. For example, Physical and Health Education Canada [12] explains that "physical literacy is a journey upon which children and youth, and everyone, develop the knowledge, skills, and attitudes they need to enable them to participate in a wide variety of activities". The Australian Sports Commission [13] also emphasizes physical literacy as a lifelong holistic learning gained and applies in the context of movement and physical activity, by integrating physical, psychological, social and cognitive abilities.

The clarity of the concept underlying physical literacy and holistic formulation of definitions has stimulated experts, researchers, practitioners, and policymakers in the world to promote, develop, and formulate their own physical literacy models in accordance with the context and culture of physical education and sports in their country. Research from Keegan et al. [14] has successfully developed a standard physical literacy model and framework that fits the context of physical education in Australia. Barnett et al. [15] also designed guidelines for the selection of physical literacy measures in physical education in Australia. In other countries, research from Wainwright, Goodway, Whitehead, Williams, and Kirk [16] has established the foundations of physical literacy in physical education in Wales as an effort to develop children's physical literacy. The massive and significant development of physical literacy in various developed countries in the world has stimulated countries in Asia, such as Singapore, China, and India to formulate their own physical literacy programs [17]. Physical literacy also provides a new perspective to better understand the benefits of physical activity for life and health [17], [18], as well as an effort to improve the quality of physical education, learning, teachers and learners [19].

In Indonesia, the discussion about physical literacy is still very limited and there is no program which focuses on developing the concept of physical literacy. Literature in international journals still does not exist, only a few articles from academics/researchers that discuss in general about the concept and promotion of physical literacy. The lack of empirical studies and research on physical literacy conducted in Indonesia, led to a lack of accessible literature as an initial reference to conduct scientific research. It can also illustrate that most of the researchers, experts, practitioners, and stakeholders related in the field of physical education, sports, health, and recreation in Indonesia, still have not explored, studied/researched, and operationalized the concept of physical literacy.

Another problem which became the focus of this study is related to the culture of physical education in elementary schools, especially in West Nusa Tenggara, Indonesia. The results of research from Irmansyah, Sakti, Syarifoeddin, Lubis, and Mujriah [20] emphasized that there are still far-right problems and gaps about the praxis of physical education learning in elementary schools. There is still no coherence between theory and practice, curriculum formulation has not been fully implemented properly, the demands of policymakers who are still oriented towards sports achievement, and the teacher's lack of understanding of the concepts, models, and assessments of physical education learning. These findings further reinforce the vulnerability of the loss of the existence of physical education, as well as justify the benefits of physical education learning in elementary schools [21]. Therefore, these results should be considered for experts to offer solutions through scientific research, and policymakers to plan and reformulate the programs or curriculum arrangements of physical education that are considered established, to be more focused on the urgent needs in the field [22].

Based on the explanation of the lack of empirical research on physical literacy and the complexity of problems in physical education in elementary school, a conceptual model with a holistic approach in physical education learning is needed that is able to offer new perspectives and provide constructive solutions in accordance with the needs and characteristics of elementary school students. Kirk [23] also emphasized that physical education has the potential to provide various educational benefits for learners, so that the improvement of the quality of physical education learning must be done by continuing to explore, review, modify, and develop physical education learning models that suit the needs of learners. This study aims to develop a model of physical literacy in the culture of physical education in elementary schools. This model of physical literacy is 
expected to be used as an alternative reference in adjusting the competencies taught in physical education learning in elementary schools, as well as in determining assessments in accordance with the concept of physical literacy. Therefore, the formulation of research questions focused on how the construction and operationalization of the model of physical literacy in the culture of physical education in elementary schools?

\section{Materials and Methods}

\subsection{Participants}

The selection of participants in this study used purposive sampling strategies [24], because the participants must have expertise in the fields of physical education and sports, physical literacy, and development of elementary school-age children. The process of selecting experts is carried out prior to data collection, and is determined by analyzing the educational and scientific background of experts to obtain conformity with the theme and objectives of research. After determining the names of the experts, the researchers then proposed to the Graduate Schools, Yogyakarta State University, to issue an official license in the form of an expert approval sheet in the research stage. A total of $(n=20)$ experts approved or agreed to be participants in the study, which included 14 men and 6 women.

The experts who have been selected represent their respective regions, namely West Nusa Tenggara $(n=13)$ and Special Region of Yogyakarta $(n=7)$. Both regions and provinces are part of Indonesia. In particular, 8 participants were academics who focused on physical education and sports, 2 participants were stakeholders who focused their work to provide education and training related to physical education learning, and 10 participants were professional physical education teachers who had certificates of teacher profession and on average had experience teaching physical education for 11.3 years. Description of the characteristics of experts and practitioners who are willing to follow the research, can be seen in (Table 1).

Table 1. Characteristic of Experts and Practitioners

\begin{tabular}{|c|c|c|c|}
\hline Category & Description & Frequency & Commentary \\
\hline \multirow{2}{*}{ Gender } & Male & 14 & Participant \\
\hline & Female & 6 & Participant \\
\hline \multirow{2}{*}{ Age (Years) } & Average & 45.8 & Years \\
\hline & Range & $27-65$ & Years \\
\hline \multirow{2}{*}{ Location } & West Nusa Tenggara, Indonesia & 13 & Country \\
\hline & Special Region of Yogyakarta, Indonesia & 7 & Country \\
\hline \multirow{13}{*}{ Area of Expertise } & Sports science & 5 & Expert \\
\hline & Physical education and sport & 14 & Expert \\
\hline & Sport pedagogy & 5 & Expert \\
\hline & Sport psychology & 2 & Expert \\
\hline & Sport medicine & 4 & Expert \\
\hline & Sport coaching & 4 & Expert \\
\hline & Children and youth sport & 7 & Expert \\
\hline & Assessment and measurement & 4 & Expert \\
\hline & Multilateral development of children & 5 & Expert \\
\hline & Fundamental movement skill & 6 & Expert \\
\hline & Curriculum development & 2 & Expert \\
\hline & Physical education teacher & 10 & Practitioners \\
\hline & Stakeholders & 2 & Practitioners \\
\hline \multirow{3}{*}{ Career Length (Years) } & Sum & 420 & Years \\
\hline & Average & 21 & Years \\
\hline & Range & $5-42$ & Years \\
\hline
\end{tabular}




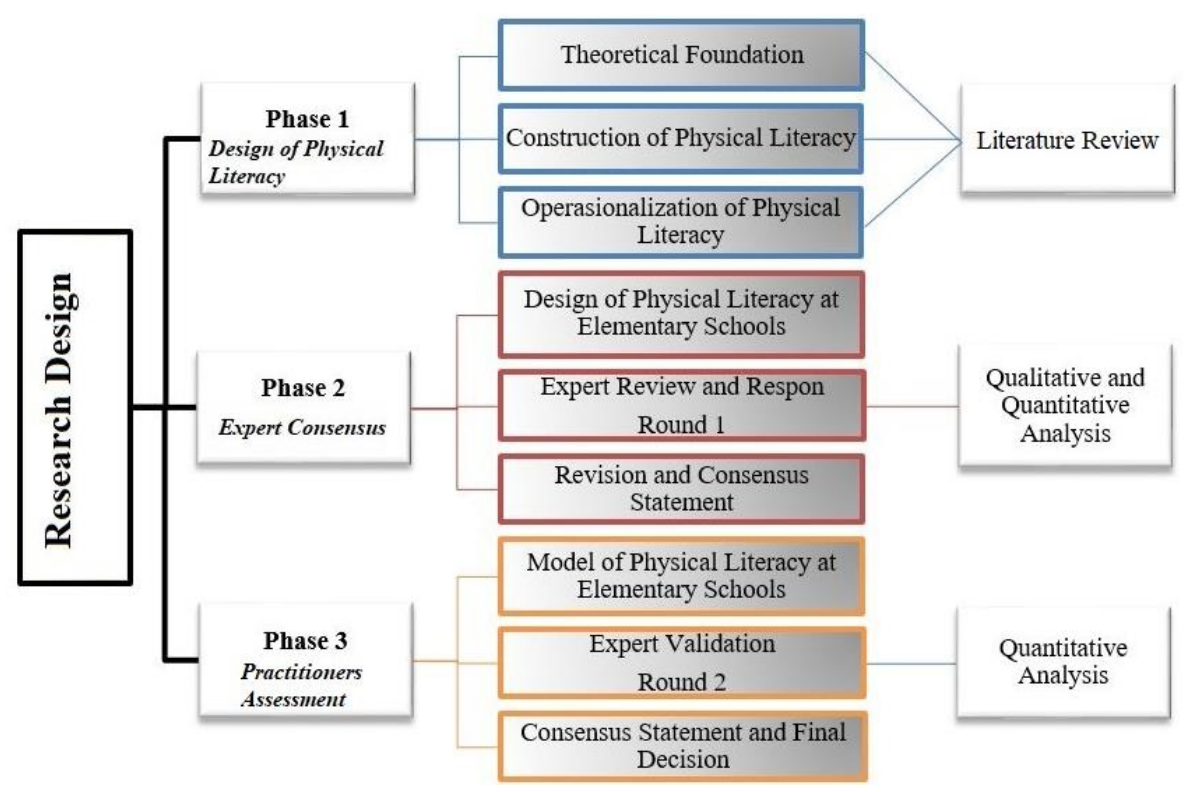

Figure 1. Flowchart of Research Design

\subsection{Research Design}

The research method uses a mixed method with sequential exploratory strategies, which focuses on qualitative data collection and analysis as primary data, quantitative data as secondary data [25]. The design of this study emphasizes the collective assessment of experts and practitioners to map the construction of physical literacy according to the culture of physical education in elementary school [26]. The procedure in this study consisted of three phases, namely: Phase 1. Design of Physical Literacy; Phase 2. Expert Consensus; and Phase 3. Practitioners Assessment, (see Figure 1).

\subsection{Data Analysis}

In Phase 1, data analysis uses descriptive qualitative [25] to map relevant literatures as the theoretical basis of the physical literacy design which is being developed. In Phase 2, data analysis also uses qualitative descriptive to obtain the results of reviews and responses of experts related to physical literacy design, and descriptive quantitative is used to obtain the percentage value of the agreement from experts. In Phase 3, descriptive quantitative analysis [27] is also used as supporting data to regain the percentage value of agreements from practitioners or teachers, as well as strengthen the study results in phases 1 and 2 .

\section{Results}

Phase 1. (Design of Physical Literacy). The researcher did the mapping of the relevant theoretical foundations in the preparation of physical literacy design. The design of physical literacy is a theoretical building on the concept of physical literacy that is adapted to the context of physical education learning in elementary school. This conceptual building is based on literature from different countries of the world related to physical literacy [1], physical education [28], 2013 curriculum in Indonesia, and assessment or measurement of physical literacy [29], [30]. Design of physical literacy results covers three main themes, namely; (a) theoretical foundation, (b) construction of physical literacy, and (c) operationalization of physical literacy.

The theoretical foundation is based on the results of research from Edwards et al. [1] which has conducted a systematic review that includes 60 scientific articles related to physical literacy. In determining the theoretical basis that supports the design of physical literacy, researchers conducted a literature review on research conducted by Keegan et al. [14], namely 'Model of physical literacy construction in Australia', and Barnett et al. [15], namely 'Guidelines for the selection of physical literacy measures in physical education in Australia'. Furthermore, analysis of the construction of physical literacy produces 38 competency indicators that are divided into; 10 indicators of motivation and confidence, 12 indicators of physical competence, 7 indicators of knowledge and understanding, and 9 indicators of behavior (social). Finally, the operationalization of physical literacy using the 'Sport Education Model' based on the humanism learning theory. The sports education model aims to increase learners' interest in sports activities, develop understanding, strategies, and sports skills, and foster understanding and ethics in sports. While, humanism learning theory focuses the attention on learners, which are holistic beings who have a series of unique experiences and potentials within each other that must be facilitated in the learning process.

Phase 2. (Expert Consensus). The results of the 
physical literacy design are examined and responded to by experts $(n=8)$ who have areas of expertise related to physical literacy and physical education. Experts review the substance or overall content of the developed physical literacy design, which includes theoretical foundations, definitions, components, sub-components, and assessments of physical literacy. After obtaining the results of the review from experts, the researchers then conducted an open ended interview [25] to further explore the opinions and responses of experts to the design of physical literacy. The results of interviews and responses from experts, became the material in making revisions to improve the quality of content of physical literacy design. Consensus statements are also made at this phase to obtain the validity of the study from experts. The percentage value agreed in this study should reach more than $80 \%$ [14].

The results of reviews and responses from experts $(\mathrm{n}=$ 8 ), explained that the design of physical literacy still has some shortcomings, such as; substance, material, and objectives of physical literacy, interrelation between physical literacy and physical education, explanation of the relationship between the theory used, mapping of components and sub-components of physical literacy, and assessment of physical literacy must be adjusted to the characteristics of elementary school students in Indonesia. These results show that the design that researchers have formulated still needs a lot of improvement in terms of substance or content of physical literacy design in physical education in elementary school. However, experts have reached an agreement that the design of physical literacy developed, as a whole, is closely in line with the culture of physical education in elementary schools. This is evidenced by the validation of experts who achieved a percentage value of the deal of $86.01 \%$.

Phase 3. (Practitioners Assessment). After doing review and validation of experts in phase 2, the design of physical literacy above, has been transformed into a model of physical literacy that has quality content relevant to the theoretical foundation and needs in the field. However, in order to obtain a model of physical literacy in accordance with the practice of physical education learning in elementary school, further validation is required by practitioners and teachers of physical education itself. Validation is carried out by $(n=2)$ stakeholders in physical education learning, and $(n=10)$ professional physical education teachers. The assessment of the physical literacy model using a questionnaire with a 4-point Type Likert scale [31]. The results of this validation, then analyzed quantitatively to obtain the agreed percentage value, became the final model agreed by experts and researchers themselves. In phase 2 , the agreed percentage value is $80 \%$ [14], but in phase 3, the percentage value is increased to $85 \%$. This has been agreed upon by experts based on the assumption that the percentage value must increase from the previous phase to add to the validity of the developed model.
The review on this phase emphasizes the suitability of physical literacy models with physical education learning practices in elementary schools, or more likely to analyze practically the operational model of physical literacy. After conducting a review, the experts then provide statistical assessment or validation using the questionnaire that has been prepared. The validation results of experts have reached an agreement that the physical literacy model developed, is very appropriate and can be implemented in the practice of physical education learning in elementary schools. This is evidenced by the validation results of experts who achieved a percentage value of the agreement of $91.84 \%$.

\section{Discussion}

\subsection{Phase 1. Design of Physical Literacy}

\subsubsection{Theoretical Foundation}

The design of physical literacy must be adapted to the core elements underlying the concept of physical literacy, which are motivation, confidence, physical competence, knowledge and understanding [32]. In the preparation of physical literacy design in the culture of physical education in elementary school, it must include all the parts that support the creation of a design that is structured, systematic, flexible, meaningful, has a clear purpose, and applicative (easy to use) [9], [33], [34]. One of the most important supporting parts/systems in the preparation of this design is the theoretical basis on which physical literacy design is based. With a clear and relevant theoretical foundation, it can make it easier to determine the core components covered in physical literacy, and provide an overview of what indicators are contained in those core components.

In general, the understanding of physical literacy is based on the results of research from Edwards et al. [1] which has conducted a systematic review that includes 60 scientific articles related to physical literacy. This systematic review is used as a basic study in providing additional knowledge of physical literacy to experts regarding the definitions, concepts, and associations of physical literacy that are being used and developed around the world. Furthermore, in determining the theoretical basis that supports the design of physical literacy, researchers conducted a literature review on research conducted by Keegan et al. [14], namely 'Model of physical literacy construction in Australia'. This model has mapped out variables that support the core components of physical literacy in accordance with the context of physical education and sport in Australia, and provided practical guidance on the operationalization and measuring of physical literacy in the physical education learning process [15]. Other literature that supports the design of physical literacy is related to physical education 
in elementary schools [28], and government policies contained in the physical education curriculum in Indonesia.

Furthermore, in assessing physical literacy, researchers used physical literacy assessments from Canada that have provided practical instructions in assessing/measuring each variable in the core components of physical literacy [29], [35], and Physical Fitness Test of Indonesia (PFTI) to determine the physical fitness level of elementary school students [30]. The entire theoretical foundation is tailored to the core competencies that must be achieved in physical education learning or curriculum 2013 in Indonesia.

\subsubsection{Construction of Physical Literacy}

Another important factor in the preparation of physical literacy design is the physical education learning model that is used as a media in achieving learning objectives. The content that must exist in the learning model is based on Joyce, Weil, and Calhoun [36] theory, i.e. focus, syntax, social system, reaction principle, support system, and application/impact model. Focus emphasizes on the pedagogical approach used, namely the sport education model and the humanism learning theory. Syntax explains the stages of learning and how the model develops as needed. Social system emphasizes the interaction between learners, expected norms, and behaviors of learners that must be appreciated. Reaction principle emphasizes the behavior of teachers in responding to the diversity of student characteristics. Support system includes all forms of resources that support the learning process. Application explains the impact of implementing developed models.

Based on the theoretical basis and objectives of the design of physical literacy above, 38 competency indicators are obtained that are divided into; 10 indicators of motivation and confidence, 12 indicators of physical competence, 7 indicators of knowledge and understanding, and 9 indicators of behavior (social). All indicators of physical literacy competency will be validated by experts, in accordance with the context of physical education learning in elementary schools. Here's the design of physical literacy in the culture of physical education in elementary schools that has been formulated in (Figure 2).

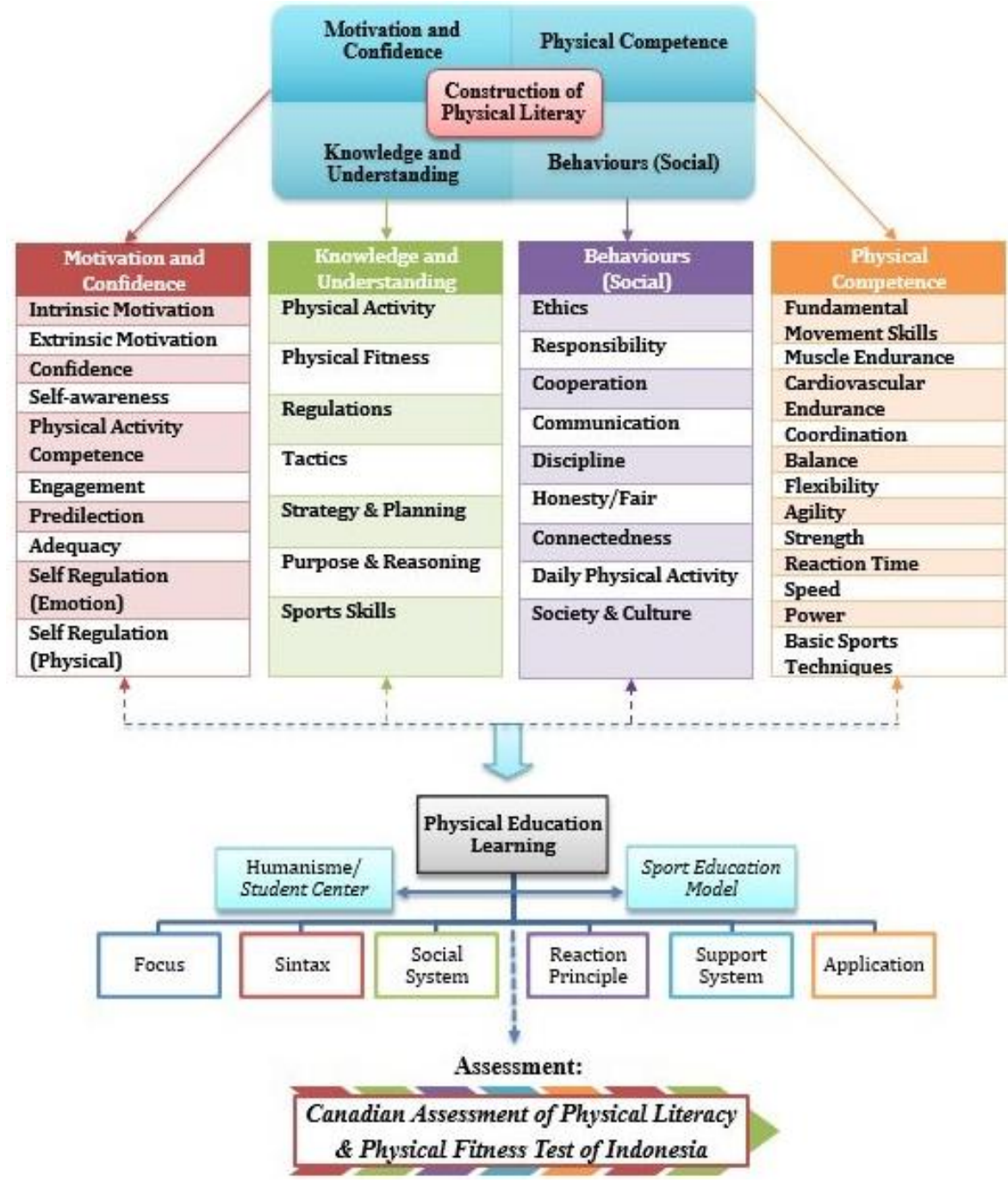

Figure 2. Model of Physical Literacy in the Culture of Physical Education in Elementary Schools 


\subsubsection{Operationalization of Physical Literacy}

Operationalization of physical literacy is a practical form of the application of physical literacy through physical education learning in elementary schools. The physical education learning model used was the 'Sport Education Model' [37] based on the humanism learning theory [38]. The sport education model is described as a pedagogical model designed to provide an authentic and educationally rich sports experience for girls and boys in the context of physical education learning [37]. The sports education model has six main characteristics, which stem from how sport is conducted in a community and inter-school context. These characteristics include; seasons, affiliations, schedule of competitions, culminating event, recording, and festivals [39], which aim to increase learners' interest in sports activities, develop understanding, strategies, and sports skills, and foster understanding and ethics in sports. Sports education is also considered more successful in maintaining intrinsic motivation levels, task orientation, climate mastery (environment), cooperation, and social interaction of learners [40].

The relationship and relevance between sports education models and physical literacy have been discussed in much of the scientific literature. For example, papers from Durden-Myers, Green, and Whitehead [41] propose that the sports education model should be an important part of the physical education curriculum and as one of the models for promoting physical literacy, as this model can give learners ample opportunities to work with others in a variety of roles, so that learners learn to take personal and social responsibility. This paper is relevant to a review from Flemons, Diffey, and Cunliffe [42] which explains some useful pedagogical approaches in promoting aspects of physical literacy. One of them is the approach of sports education. Sports education approaches can foster personal and social responsibility [43], provide knowledge and understanding of health-related fitness [44], and various forms of play can help learners to gain a deeper understanding of playing activities [45].

Furthermore, the humanism learning theory focuses the attention on learners, which should be seen as the place where attention about learning begins. Learners are holistic beings who have a series of unique experiences and potentials within each other that must be facilitated in the learning process [5], [7], [46], so as to fulfill their potential and achieve self-actualization [38]. Meanwhile, in physical literacy it is explained that humanism learning theory in particular has a conformity with physical literacy to promote the learning and development of physical literacy of learners, because humanism learning theory places learners as the center of learning, as well as a strong emphasis on the management/regulation of emotions (psychological) [47].

\subsection{Phase 2. Expert Consensus}

The results of expert validation on the design of physical literacy in the culture of physical education in elementary schools are formulated in Table 2.

Table 2. Expert Validation in Phase 2 (Expert Consensus)

\begin{tabular}{ccccc}
\hline Expert & Number of Question & Total Score & Percentage \% & Category \\
\hline Expert 1 & 40 & 134 & 83,75 & Valid \\
Expert 2 & 40 & 139 & 86,87 & Valid \\
Expert 3 & 40 & 129 & 80,62 & Valid \\
Expert 4 & 40 & 132 & 82,50 & Valid \\
Expert 5 & 40 & 147 & 91,87 & Valid \\
Expert 6 & 40 & 146 & 91,25 & Not Valid \\
Expert 7 & 40 & 126 & 78,75 & 92,50 \\
Expert 8 & 40 & 148 & & Valid \\
\end{tabular}

Note. The percentage value of the agreement in Phase 2 must be more than (> 80\%) with criteria of "valid' and less than $(<80 \%)$ "invalid" criteria. 
These results emphasize that the physical literacy design developed has a high degree of validity, so it is feasible to be implemented in the field or in the process of learning physical education. This process of assessment or validation of physical literacy is also relevant to some of the results of the development of physical literacy models in several countries, most of experts in assessing the physical literacy model in their countries through Delphi studies [14], [26], [31], [48] or by mixed methods [49], [50]. However, the results of this quantitative analysis, can not be used as the only basis in justifying the validity of physical literacy design, it takes review and responses textually or verbally from experts to obtain a more credible validity results, both in content/main idea, construction, application, and assessment. This is because the design of physical literacy developed has structures or parts that have never been discussed in physical education learning in elementary school, thus it must be given a review directly by experts.

The results of reviews and responses from experts, explaining that the design of physical literacy still has some shortcomings, among others; (a) there are too many structures or parts in the design of physical literacy; (b) additional discussions related to concepts, materials, and objectives of physical literacy; (c) explanation of the interrelation between physical literacy and physical education; (d) more detailed explanation of the relationship between the theories used; (e) the design of physical literacy must be tailored to the characteristics of primary school learners; (f) explanation of physical literacy components and indicators for which students are assessed; (g) the learning model should be practical and easy for teachers to understand, more varied, and include aspects of attitudes, knowledge, and skills; and (h) the physical literacy assessment model is tailored to the characteristics of elementary school students in Indonesia. There are many shortcomings of the expert review, explaining that the design of physical literacy developed still needs to be revised to produce a design that has quality and deserves to be implemented in the process of physical education learning in elementary school.

These results further explain that the concept of physical literacy [5] which is developing in several countries [17] and is the focus of studies by experts in the fields of physical education, sports, health, recreation, even coaching or long-term athlete development [17], has complexity if formulated into a model that suits the environment or culture of a country. This is due to the holistic nature underlying the concept of physical literacy [4] or philosophical foundations that emphasize the philosophy of monism, existentialism, and phenomenology [46]. Experts in any country must be able to truly identify, understand, analyze and map the concept of physical literacy if they want to integrate physical literacy in the settings of physical education and sports in their country.

\subsection{Practitioners Assessment}

The results of expert validation of the model of physical literacy in the culture of physical education in elementary school, are formulated in (Figure 3).

These results are not obtained instantaneously or simply provide normative assessment by practitioners or teachers, but rather through a planned, structured, and systematic physical education learning process [51], [52] in accordance with the developed physical literacy model. Technically, before assessing the physical literacy model, previously practitioners or teachers are given an overview of the physical literacy model developed and how to implement it in the physical education learning process through group discussions [24]. After a group discussion, practitioners or teachers are given time to study the physical literacy model developed, in order to strengthen and improve theoretical knowledge and understanding [53].

The results of the teacher's reflection also explained that after the application of physical literacy models in physical education learning, learners are more motivated, confident, fun, and enjoyed when they are asked to perform movements, physical activities, sports techniques, games, or exercises. These results correspond to the motivation and confidence component in the concept of physical literacy, which explains that motivation and confidence are the foundations and core components in developing individual physical literacy [3], [7]. In addition, the results of research from Liu, Xiang, Lee, and $\mathrm{Li}$ [54] also explained that a well-prepared learning environment by teachers, can have an impact on increasing motivation and confidence or indirectly lead to the development of physical literacy of learners. This finding is also very essential because it is justifying the benefits of physical education in providing meaningful education and developing the potential of learners as a whole [20], it can be slightly treated with the spirit and excitement of learners in following physical education learning. Therefore, all physical education learning materials transferred to learners must always emphasize learning that is innovative, fun, enjoyable, and has an element of friendship [23], [55]. 


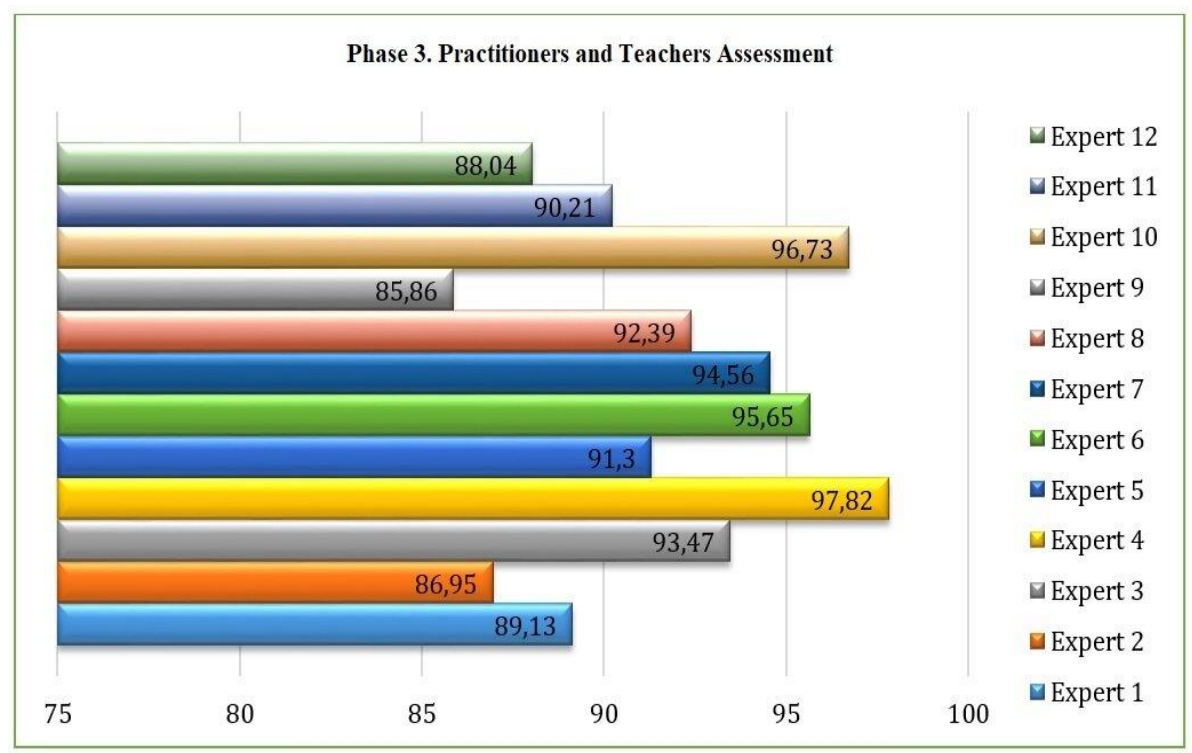

Figure 3. Flowchart of Expert Validation in Phase 3 (Practitioners Assessment)

\section{Conclusions}

The development of physical literacy models in the culture of physical education in elementary schools, can be concluded to have theoretical and practical success, because the validation results from experts show a percentage of value that exceeds the statement of agreement, namely $86.01 \%$ (Phase 2) and $91.84 \%$ (Phase $3)$. The results of the expert review also showed the suitability of the substance of the physical literacy model developed with the culture of physical education in elementary schools, as well as the characteristics and needs of learners. The development of this physical literacy model is expected to be an initial reference for academics, researchers, students, and policy makers related to the concept of physical literacy in Indonesia, especially in elementary school education units. These results can also serve as teaching and training guidelines for teachers and practitioners, to improve the quality of physical education learning, teacher knowledge and understanding, and potential development of learners. Although, this study shows convincing results and is relevant to the culture of physical education in elementary school, however, there are still many limitations in this study, such as; limited number of experts used, research locations focusing only in two area of Indonesia, implementation of physical literacy model has not been carried out to the maximum in the process of physical education learning, and the form of physical literacy assessment has not been tested on teachers and learners.

Future research is expected to build and review the results of this research from various perspectives, conduct in-depth exploration and study of the concept of physical literacy in accordance with local wisdom and physical education culture in the region, and adapt to various innovations of learning and traditional sports that become the cultural identity of the Indonesian nation. In practical terms, this model of physical literacy should be tested and evaluated to determine the validity, effectiveness, and usefulness or benefits of its application. These results can also still be debated and reviewed to assess whether the perspectives offered in this study can be transferred and implemented into cultures or contexts other than physical education, such as; sports health, sports recreation, sports coaching, social inclusion, gender equality in sports, global citizenship education, and others.

\section{Acknowledgements}

The author would like to thank the Promoters of the Dissertation for assistance and guidance during the dissertation completion process. Promoters have a huge contribution in supporting this research, so the author rewards it by including it as Co-authors.

\section{Conflict of Interest}

The author declares that he has no conflict of interests.

\section{REFERENCES}

[1] L. C. Edwards, A. S. Bryant, R. J. Keegan, K. Morgan, and A. M. Jones, "Definitions, foundations and associations of physical literacy: A systematic review," Sport. Med., vol. 47, no. 1, pp. 113-126, Jan. 2017.

[2] D. Dudley, J. Cairney, N. Wainwright, D. Kriellaars, and D. Mitchell, "Critical considerations for physical literacy policy in public health, recreation, sport, and education 
agencies," Quest, vol. 69, no. 4, pp. 436-452, Oct. 2017.

[3] M. Whitehead, "Motivation and the significance of physical literacy for every individual," in Physical literacy: Throughout the lifecourse, M. Whitehead, Ed. London, UK: Routledge, 2010, pp. 30-43.

[4] L. Young, J. O’Connor, and L. Alfrey, "Physical literacy: A concept analysis," Sport. Educ. Soc., vol. 25, no. 8, pp. 946-959, 2020.

[5] M. Whitehead, "The concept of physical literacy," in Physical literacy: Throughout the lifecourse, M. Whitehead, Ed. London, UK: Routledge, 2010, pp. 10-20.

[6] M. Whitehead, "The concept of physical literacy," Eur. J. Phys. Educ., vol. 6, no. 2, pp. 127-138, Jan. 2001.

[7] E. J. Durden-Myers, M. Whitehead, and N. Pot, "Physical literacy and human flourishing," J. Teach. Phys. Educ., vol. 37, no. 3, pp. 308-311, Jul. 2018.

[8] P. Jurbala, "What Is physical literacy, really?," Quest, vol. 67, no. 4, pp. 367-383, Oct. 2015.

[9] S. Lundvall, "Physical literacy in the field of physical education - A challenge and a possibility," J. Sport Heal. Sci., vol. 4, no. 2, pp. 113-118, Jun. 2015.

[10] M. Whitehead, "Definition of physical literacy and clarification of related issues," ICSSPE J. Sport Sci. Phys. Educ., vol. 65, pp. 29-34, 2013.

[11] L. C. Edwards, A. S. Bryant, R. J. Keegan, K. Morgan, S.-M. Cooper, and A. M. Jones, "Measuring' physical literacy and related constructs: A systematic review of empirical findings," Sport. Med., vol. 48, no. 3, pp. 659682, Mar. 2018.

[12] Physical and Health Education (PHE) Canada, "Physical literacy," 2019. [Online]. Available: https://phecanada.ca/a ctivate/physical-literacy.

[13] Australian Sports Commission, "Australian physical literacy framework versi 2," 2019. [Online]. Available: https://www.sportaus.gov.au/_data/assets/pdf_file/0019/7 10173/35455_Physical-Literacy-Framework_access.pdf.

[14] R. J. Keegan et al., "Defining physical literacy for application in Australia: A modified delphi method," J. Teach. Phys. Educ., vol. 38, no. 2, pp. 105-118, Apr. 2019.

[15] L. M. Barnett et al., "Guidelines for the selection of physical literacy measures in physical education in Australia," J. Teach. Phys. Educ., vol. 38, no. 2, pp. 119125, Apr. 2019.

[16] N. Wainwright, J. Goodway, M. Whitehead, A. Williams, and D. Kirk, "Laying the foundations for physical literacy in Wales: The contribution of the Foundation Phase to the development of physical literacy," Phys. Educ. Sport Pedagog., vol. 23, no. 4, pp. 431-444, Jul. 2018.

[17] C. Shearer et al., "How is physical literacy defined? A contemporary update," J. Teach. Phys. Educ., vol. 37, no. 3, pp. 237-245, Jul. 2018.

[18] P. E. Longmuir and M. S. Tremblay, "Top 10 research questions related to physical literacy," Res. Q. Exerc. Sport, vol. 87 , no. 1 , pp. 28-35, Jan. 2016.
[19] UNESCO, Quality physical education (QPE): Guidelines for policy-maker. France: United Nations Educational, Scientific and Cultural Organization (UNESCO), 2015.

[20] J. Irmansyah, N. W. P. Sakti, E. W. Syarifoeddin, M. R. Lubis, and Mujriah, "Physical education, sports, and health in elementary schools: Description of problems, urgency, and understanding of teacher perspectives," J. Pendidik. Jasm. Indones., vol. 16, no. 2, pp. 115-131, 2020.

[21] J. Irmansyah, R. Lumintuarso, F. Sugiyanto, and P. Sukoco, "Children's social skills through traditional sport games in primary schools," Cakrawala Pendidik., vol. 39, no. 1, pp. 39-53, Feb. 2020.

[22] K. J. Yi et al., "Future directions for physical literacy education: Community perspectives," J. Phys. Educ. Sport, vol. 20, no. 1, pp. 123-130, 2020.

[23] D. Kirk, "Educational value and models-based practice in physical education," Educ. Philos. Theory, vol. 45, no. 9, pp. 973-986, Sep. 2013.

[24] I. Jones, Research methods for sports studies, 3rd ed. Milton Park, Abingdon, Oxon, New York, NY: Routledge, 2015.

[25] J. W. Creswell and C. N. Poth, Qualitative inquiry and research design: Choosing among five approaches, 4th ed. Thousand Oaks, California: SAGE Publications Inc, 2018.

[26] C. A. Shortt, C. A. Webster, R. J. Keegan, C. A. Egan, and A. S. Brian, "Operationally conceptualizing physical literacy: Results of a delphi study," J. Teach. Phys. Educ., vol. 38, no. 2, pp. 91-104, Apr. 2019.

[27] A. J. Onwuegbuzie and K. M. T. Collins, "The role of sampling in mixed methods-research," KZfSS Kölner Zeitschrift für Soziologie und Sozialpsychologie, vol. 69, no. S2, pp. 133-156, Oct. 2017.

[28] R. P. Pangrazi and A. Beighle, Dynamic physical education for elementary school children, 18th ed. United States of America: Pearson Education, Inc, 2016.

[29] Canadian Assessment of Physical Literacy, Manual for test administration, 2nd ed. Ontario, Canada: Healthy Active Living and Obesity Research Group, 2017.

[30] Y. Sukarmin and Sudardiyono, "Physical fitness profiles of lower-level students in elementary schools based on observation guidelines," J. Phys. Educ. Sport, vol. 17, no. 1, pp. 84-91, 2017.

[31] C. E. Francis et al., "The Canadian Assessment of Physical literacy: Development of a model of children's capacity for a healthy, active lifestyle through a Delphi process," J. Phys. Act. Heal., vol. 13, no. 2, pp. 214-222, 2016.

[32] M. Whitehead, "The philosophical underpinning of the concept of physical literacy," in Physical literacy: Throughout the lifecourse, M. Whitehead, Ed. London, UK: Routledge, 2010, pp. 21-29.

[33] D. M. Castelli, J. M. Barcelona, and L. Bryant, "Contextualizing physical literacy in the school environment: The challenges," J. Sport Heal. Sci., vol. 4, no. 2 , pp. 156-163, Jun. 2015.

[34] S. Silverman and K. Mercier, "Teaching for physical literacy: Implications to instructional design and PETE," J. Sport Heal. Sci., vol. 4, no. 2, pp. 150-155, Jun. 2015. 
[35] P. E. Longmuir et al., "Canadian assessment of physical literacy second edition: A streamlined assessment of the capacity for physical activity among children 8 to 12 years of age," BMC Public Health, vol. 18, no. S2, p. 1047, Oct. 2018.

[36] B. R. Joyce, M. Weil, and E. Calhoun, Models of teaching, 9th ed. Boston: Pearson, 2015.

[37] D. Siedentop, P. A. Hastie, and H. Van der Mars, Complete guide to sport education, 3rd ed. Champaign, IL: Human Kinetics, 2019.

[38] C. R. Rogers, "Toward a theory of creativity," ETC A Rev. Gen. Semant., vol. 11, no. 4, pp. 249-260, 2019.

[39] P. A. Hastie and T. L. Wallhead, "Operationalizing physical literacy through sport education," J. Sport Heal. Sci., vol. 4, no. 2, pp. 132-138, Jun. 2015.

[40] T. L. Wallhead, A. C. Garn, and C. Vidoni, "Sport education and social goals in physical education: Relationships with enjoyment, relatedness, and leisure-time physical activity," Phys. Educ. Sport Pedagog., vol. 18, no. 4, pp. 427-441, Sep. 2013.

[41] E. J. Durden-Myers, N. R. Green, and M. Whitehead, "Implications for promoting physical literacy," J. Teach. Phys. Educ., vol. 37, no. 3, pp. 262-271, Jul. 2018.

[42] M. Flemons, F. Diffey, and D. Cunliffe, "The role of PETE in developing and sustaining physical literacy informed practitioners," J. Teach. Phys. Educ., vol. 37, no. 3, pp. 299-307, Jul. 2018.

[43] D. Hellison, Teaching personal and social responsibility through physical activity, 3rd ed. United States of America: Human Kinetics, 2011.

[44] A. Chen, "Operationalizing physical literacy for learners: Embodying the motivation to move," J. Sport Heal. Sci., vol. 4, no. 2, pp. 125-131, Jun. 2015.

[45] J. Mandigo, K. Lodewyk, and J. Tredway, "Examining the impact of a teaching games for understanding approach on the development of physical literacy using the passport for life assessment tool," J. Teach. Phys. Educ., vol. 38, no. 2, pp. 136-145, Apr. 2019.
[46] N. Pot, M. Whitehead, and E. J. Durden-Myers, "Physical literacy from philosophy to practice," J. Teach. Phys. Educ., vol. 37, no. 3, pp. 246-251, Jul. 2018.

[47] N. R. Green, W. M. Roberts, D. Sheehan, and R. J. Keegan, "Charting physical literacy journeys within physical education settings," J. Teach. Phys. Educ., vol. 37, no. 3, pp. 272-279, Jul. 2018.

[48] G. R. Jones et al., "Development of a physical literacy model for older adults - A consensus process by the collaborative working group on physical literacy for older Canadians," BMC Geriatr., vol. 18, no. 1, pp. 1-16, 2018.

[49] N. Wainwright, J. Goodway, M. Whitehead, A. Williams, and D. Kirk, "The foundation phase in Wales - A play-based curriculum that supports the development of physical literacy," Educ. 3-13, vol. 44, no. 5, pp. 513-524, 2016.

[50] J. J. Scott, S. Hill, D. Barwood, and D. Penney, "Physical literacy and policy alignment in sport and education in Australia," Eur. Phys. Educ. Rev., vol. 27, no. 2, pp. 328 347, May 2021.

[51] H. Sun, W. Li, and B. Shen, "Learning in physical education: A self-determination theory perspective," J. Teach. Phys. Educ., vol. 36, no. 3, pp. 277-291, Jul. 2017.

[52] P. Legrain, G. Escalié, L. Lafont, and S. Chaliès, "Cooperative learning: A relevant instructional model for physical education pre-service teacher training?," Phys. Educ. Sport Pedagog., vol. 24, no. 1, pp. 73-86, Jan. 2019.

[53] L. C. Edwards, A. S. Bryant, K. Morgan, S.-M. Cooper, A. M. Jones, and R. J. Keegan, "A professional development program to enhance primary school teachers' knowledge and operationalization of physical literacy," J. Teach. Phys. Educ., vol. 38, no. 2, pp. 126-135, Apr. 2019.

[54] J. Liu, P. Xiang, J. Lee, and W. Li, "Developing physically literacy in K-12 physical education through achievement goal theory," J. Teach. Phys. Educ., vol. 36, no. 3, pp. 292-302, Jul. 2017.

[55] S. Beni, T. Fletcher, and D. Ní Chróinín, "Meaningful experiences in physical education and youth sport: A review of the literature," Quest, vol. 69, no. 3, pp. 291-312, Jul. 2017. 